\title{
Two Human-Centered Approaches to Health Informatics: Cognitive Systems Engineering and Usability
}

\author{
Tania Xiao ${ }^{1,2}$, Wendy Broxham ${ }^{1}$, Cara Stitzlein ${ }^{2}$, \\ Jasmine Croll $^{1}$, and Penelope Sanderson ${ }^{1,2}$ \\ ${ }^{1}$ National ICT Australia, St Lucia, \\ Queensland, Australia \\ ${ }^{2}$ The University of Queensland, St Lucia, \\ Queensland, Australia \\ \{Penelope.Sanderson, Tania.Xiao, Wendy.Broxham, \\ Jasmine.Croll, firstname. lastname\} @nicta.com.au, \\ cara.stitzlein@uqconnect.edu.au
}

\begin{abstract}
There is growing recognition among many healthcare researchers that a human-centered approach to the design and evaluation of health information systems is vital for the success of such systems in healthcare. In this paper, we survey the work of two human-centered research communities that have been active in the area of health information systems research but that have not been adequately discussed in past comparative reviews. They are cognitive systems engineering and usability. We briefly consider the origins and contributions of the two research communities and then discuss the similarities and differences between them on several topics relevant to health information systems. Our objective is to clarify the distinction between the two communities and to help future researchers make more informed decisions about the approaches and methods that will meet their needs.
\end{abstract}

Keywords: health information systems, human-centered technology, cognitive systems engineering, usability, cognitive engineering.

\section{Introduction}

The Institute of Medicine's report "Crossing the quality chasm" (2001) has generated great interest in the development of health information systems to support safe and effective healthcare. Unfortunately, there has been limited success associated with the introduction of health information systems in healthcare settings [1]. There is growing recognition amongst many healthcare technology researchers that the failure of many health information systems can be attributed to poor understanding of the needs and work practices of the human user and the socio-technical context in which work is done [2].

There is already much research focused on human-machine interaction from a human-centered perspective. For example, Hoffman and Militello [3] identify eight 
'communities of practice' that emerged in the 1980s driven by the common goal to better understand and support human cognitive tasks. In the healthcare context, Greenhalgh et al. [2] classified existing human-centered research into nine metanarratives which include 'computer supported cooperative work', 'actor-network analyses' and 'critical sociology'.

Greenhalgh et al.'s [2] review offers new insights into human-centered health information systems research. However, because they limited their focus to Electronic Patient Records (EPR), they may have overlooked traditions that have done less research on EPRs and more research on other types of health information systems. In this paper, we extend Greenhalgh et al.'s work by discussing two research communities that have also made important contributions to our understanding of the needs and practices of the human user.

\subsection{Current Paper}

In this paper we highlight the work of the cognitive systems engineering and usability communities, neither of which was covered in Greenhalgh et al.'s [2] review. Based on our interpretation of the literature and our participation from time to time in these communities, we identify and discuss similarities and differences between them. Our goal is to bring these two communities to the attention of health informatics researchers and to clarify actual or potential confusions that may exist in the literature.

To achieve this aim, first we describe how we identified some key papers that represent the core ideas of the two traditions. Then we discuss each tradition in detail, considering their similarities and differences. Finally we note current and potential contributions of each community to the successful development and implementation of health information systems.

\section{Method}

We conducted a systematic and comprehensive search of the Web of Science (via ISI Web of Knowledge) between January and May, 2009 to investigate which research communities were involved in health information systems research. Combinations of keywords including patient safety, technology, decision support, usability, health information systems and automation were used to conduct an extensive literature search of technology in healthcare. Three researchers read through the abstracts of 1642 papers and identified four contributing research communities. We focus on two research communities, usability and CSE, for the purposes of this paper.

To refine our search to the key papers in each of the above two communities, we selected papers (1995-2009) with ten or more citations. A focused search of highlycited papers from 2006 to 2009 ensured we did not miss important recent contributions. This process resulted in approximately 25 papers for each community.

Five group sessions were conducted during which the papers were discussed in terms of their representativeness of a research community and the quality of the empirical research. As a result of the discussions, we chose to exclude certain papers to achieve a more coherent message for our paper. First, we focused on empirical work because we were interested in the application of theoretical ideas to practice. 
Second, we limited our scope to hospital-based work because it represented the dominant research context. Third, we excluded all conference papers because we wanted to focus on journal publications in which authors could present more information about their research. For the usability and CSE communities, the above exclusion process yielded a total of 7 and 8 papers respectively that we considered most representative of each area Finally, an examination of the reference lists of these empirical papers and communications with experts in the areas helped us to identify key theoretical papers that define the historical roots and major contributions of the two research communities.

\section{Results}

\subsection{Usability}

Background. Usability is defined as "the extent to which a product can be used by specified users to achieve specified goals with effectiveness, efficiency and satisfaction in a specified context of use"[4]. Fundamentally, usability professionals are concerned with enhancing the interactive experience between human users and a piece of technology (usually a computer software interface). Usability emerged as an important property of computers and computer software during the proliferation of computers in the general workplace during the 1960s and 1970s. The launch of the Macintosh in 1984 led to usability being recognized as essential to the development of computer technology [5].

The work of usability practitioners is usually informed by other research traditions including cognitive psychology, human factors and software engineering. Some of the earliest proponents of usability worked for companies such as IBM, Macintosh and Xerox PARC and later shared their experiences with the research community. In the last few decades, the usability profession has acquired a distinct identity. The Usability Professionals Association (UPA) was established in 1991 and it provides resources to support usability research and practice. In addition, the ACM-sponsored Computer Human Interaction (CHI) conference covers some advances in usability research, amongst many other topics. Moreover, publications dedicated to usability research have emerged, such as Journal of Usability Studies.

For usability professionals, the usability of a system is determined by five main criteria [6]: its learnability, efficiency, memorability, ease of recovery from errors, and user satisfaction. To achieve these criteria, usability researchers encourage (a) early user participation in terms of setting usability goals and design requirements and (b) early and iterative user testing and redesign of prototypes both in the laboratory and work context. Many sets of principles have been created to guide the development of user-centered designs [7, 8] and many techniques exist for evaluating designs, including heuristic task analysis, cognitive walkthroughs, and benchmark user testing [7, 9]. Figure 1 gives a characterization of user testing.

Usability in healthcare. In recent decades, usability has emerged as a critical issue in healthcare. Many health information systems fail because of poor usability [10]. Allwood and Kalen [11] were amongst the first to report research on health information systems inspired by usability concepts. Following a four-year field 
investigation of the introduction of a patient administration system in a Swedish hospital, they identified key issues related to the development process (such as failing to identify usability goals, not having user participation) that contributed to the difficulties users experienced when learning and using the system. More recently, many researchers have raised concerns about how poorly-designed technology may make healthcare systems more vulnerable to adverse events [12]. Thus, there is growing urgency among the usability community to use their methods to identify and prevent technology-induced error in healthcare systems [13].

Usability research in healthcare is now quite common - here we highlight some representative examples. First, researchers have used usability methods to identify associations (a) between physician order entry systems and adverse drug events [14] and (b) between handheld prescription writing tools and prescription errors [15]. Second, researchers have applied user-centered and iterative testing and design principles to the development of a health information system interface [16] and a mobile electronic patient record prototype [17]. Third, usability evaluation has been used to assist with decisions about the procurement of hospital equipment such as infusion pumps [18] and patient monitoring systems [19]. Finally, given the wide range of methods used in usability research, some research focuses on making comparisons between different usability methods to determine which method is best suited to certain evaluation needs [20].

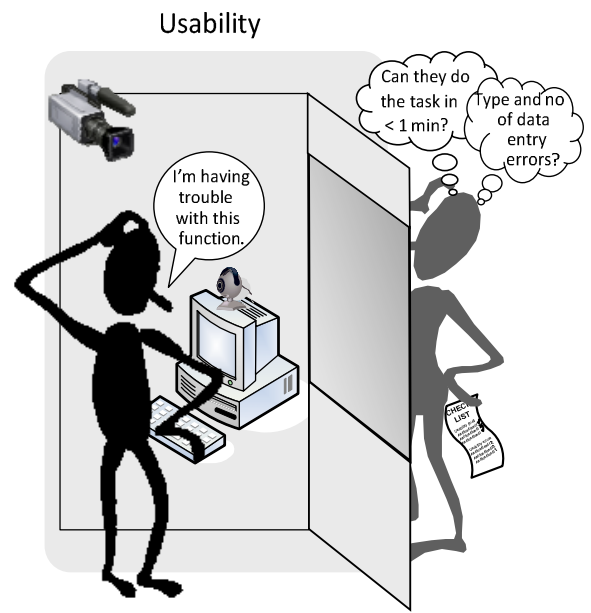

Fig. 1. Characterization of a traditional usability method (user testing). Researcher is the gray figure at right looking through a two-way mirror at a participant performing a task under controlled laboratory conditions. Thought clouds indicate representative concerns of researcher and participant.

\subsection{Cognitive Systems Engineering}

Background. Cognitive systems engineering (CSE) is an approach to the analysis, modeling, design and evaluation of complex socio-technical systems. It emerged in the early 1980s from studies of the human operator's role in process control during 
the 1960s and 1970s, culminating in analyses of the Three-Mile Island accident in 1979. The first published usage of the term "cognitive systems engineering" was in 1983 by Hollnagel and Woods [21] who argued that 'instead of viewing man-machine systems as decomposable mechanistic principles, CSE introduces the concept of a [joint] cognitive system', where man and machine work together as a team responding adaptively to complex work problems.

Many CSE researchers are human factors specialists and there is a CSE technical group within the Human Factors and Ergonomics Society. There are several journals dedicated to CSE research including the Journal of Cognitive Engineering and Decision Making journal and Cognition, Technology and Work. In addition to activity within the Human Factors and Ergonomics Society conference, there are also strong CSE communities in Europe.

CSE research is influenced by many disciplines, including systems engineering, cognitive, ecological psychology, work psychology and anthropology, and so is not considered a "discipline" by its adherents [22]. It is specifically concerned with supporting human cognitive performance in complex safety critical industries such as nuclear power, aviation and healthcare. Some CSE analysts focus on identifying constraints that operate in work domains and that shape activity [22, 23]. CSE researchers argue that if people have a good understanding of fundamental constraints in their work environment, they can reason more effectively about how to solve unfamiliar work problems. More recently, many CSE researchers have embraced a proactive approach to risk management called resilience engineering [35]. Resilience engineering focuses on (a) identifying existing work processes that help people achieve robust and adaptive performance and (b) designing systems that support those processes. Figure 2 gives a characterization of field observation with the questions that a cognitive engineer might bring to it.

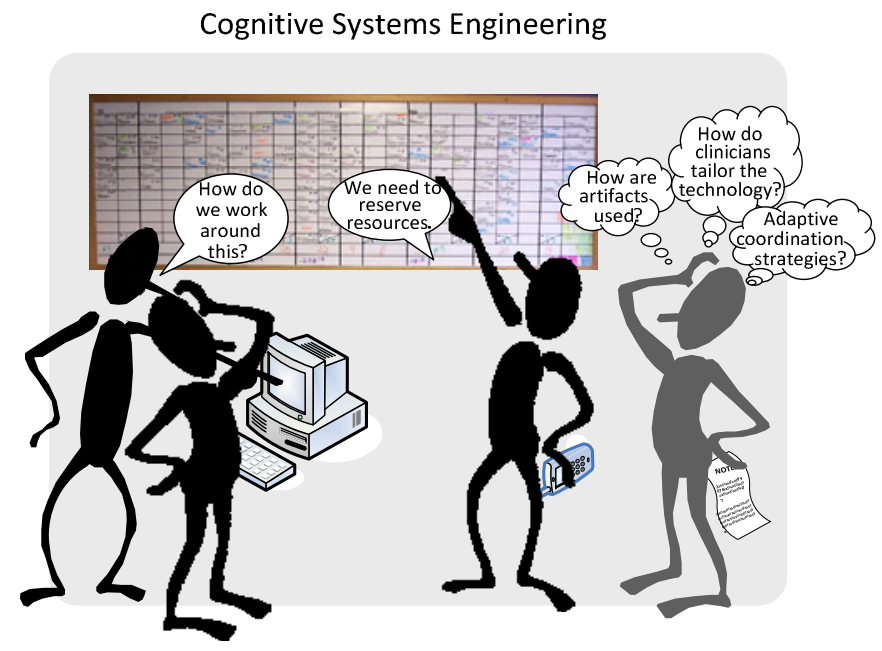

Fig. 2. Characterization of a cognitive systems engineering method (field observation). Panel in background represents a workplace artefact - a whiteboard. Figures at left are people in their workplace, whereas gray figure at right is the researcher. Thought clouds are representative questions about how work is organized and supported. 
CSE in healthcare. Because the 'computerization' of healthcare has been slower compared with other domains such as aviation, CSE research is less well established in healthcare than in other domains. An early CSE-oriented healthcare study was conducted by Gaba who investigated anaesthetists' responses to critical incidents in a simulated operating theatre environment [24]. Following the Institute of Medicine's suggestion that technology could be more effectively used in healthcare [25], CSE researchers have been quick to express concerns that the technology push might result in brittle work practices that make a system more vulnerable to failure [26].

We now highlight some key empirical works that reflect CSE concerns. First, some research [27] [28] has highlighted side effects that technology (e.g. bar code medication administration system, computerised clinical reminders) can have on communication and coordination practices that may, in turn, contribute to adverse patient outcomes.

Second, researchers have explored how the use of non-electronic cognitive artefacts (e.g. schedules and whiteboards) can reveal hidden aspects of the work that can be used to define information and usage requirements for the design of electronic support [29, 30].

Third, a few researchers have applied CSE principles to the design of health information systems including a blood antibody detection decision support system [31] and a cardiac display [32].

Finally, CSE researchers have studied how variability in and adaptation of work practices contribute to resilient and successful performance in contexts with and without technology such as the introduction of new patient monitoring systems in the operating theatre [33] or the management of bed resources in the ICU [34].

\subsection{Nuanced Distinctions between Areas}

The terms "usability", "cognitive systems engineering" and "cognitive engineering" are increasingly used in healthcare, but are sometimes confused. Here we provide our own perspective on how the terms can be distinguished and the confusions that might occur otherwise.

A term often used interchangeably with "cognitive systems engineering" is "cognitive engineering" (CE) [36]. However, for some practitioners there are important differences between CSE and CE. According to Norman, who introduced the term in 1980 and was the first person to describe CE as a unique discipline [37, 38], CE is the application of cognitive science principles to the design and evaluation of technical tools that enhance human cognitive performance in general. $\mathrm{CE}$ is typically concerned with a broader and simpler range of applications than CSE, including educational support tools and the "design of everyday things" [38, 39]. Many methods proposed by early CE researchers were relatively simple user-centered design and evaluation techniques that are now more commonly associated with usability evaluation. More recently, many $\mathrm{CE}$ researchers have performed more complex analyses using methods such as cognitive task analysis and computational cognitive modeling [40].

In a recent paper assessing usability of healthcare technology, Liljegren and Osvalder [19] referred to cognitive walkthroughs and usability tests as cognitive engineering methods. Although such methods were described as cognitive 
engineering methods in 1986 by Norman [8] this description may be less apt now for several reasons. First, cognitive walkthroughs and usability tests are now more closely associated with usability engineering or user-centered design. Second, given that cognitive systems engineering is often referred to as cognitive engineering, if cognitive walkthroughs and usability tests are referred to as cognitive engineering methods, readers might infer that they are central to CSE as well.

Given that the CSE and usability communities differ in their approach to the design and evaluation of technology, Liljegren and Osvalder's usage could conceivably lead to future researchers thinking they have adopted one approach when they are using methodologies that actually better reflect another approach. For that reason it is probably clearer if methods such as usability tests, cognitive walkthroughs, heuristic evaluations, and so on be referred to as usability engineering methods or usercentered design and evaluation methods rather than as cognitive engineering methods.

\subsection{Comparisons of CSE and Usability}

In their review of different theoretical approaches to examining the impact and effectiveness of EHRs, Greenhalgh et al. [2] presented some themes that highlight tensions among different research meta-narratives. In a similar vein, below we present five themes that show where usability and CSE approaches to healthcare may be most strongly contrasted. The first three themes are inspired by themes in the Greenhalgh et al. paper. The last two are new themes that emerged during our group discussions and that helped us distinguish between the usability and CSE communities. In Table 1 we present the studies reviewed according to which theme we think each study best represents.

Note that we do not say that the two research communities have no connection. Researchers in each community incorporate ideas from the other to strengthen their work. In addition, the two communities share many similarities: both are concerned about user experience, both are empirical in nature, and both draw from the human sciences in different forms and different ways.

The Technology. Greenhalgh et al. [2] noted two different perceptions of technology in healthcare among the human-centered research communities: (a) technology as a 'tool or container' or (b) technology as an artifact that 'acts' in the environment. Usability professionals are more likely to adopt the former view that technology is a tool that processes and communicates information [41]. Their primary concerns are to (a) determine which tasks need to be supported by the technology, and (b) design the technology and test whether it helps users effectively perform the task.

On the other hand, CSE professionals are more likely to adopt the latter view that technology is an artifact that brings new opportunities and challenges into the work environment. The primary concern is to develop new concepts or models that give designers insight into new phenomena that may emerge when new technology is introduced into a workplace (e.g. automation surprises, clumsy automation) [33, 42].

The User. Given that both are human-centered approaches, usability and CSE emphasize the importance of drawing from user experience when designing and evaluating health information systems. However, different aspects of the user are 
Table 1. List of reviewed usability and CSE studies organized according to theme comparisons

\begin{tabular}{|c|c|c|}
\hline & Usability & CSE \\
\hline The Technology & $\begin{array}{l}\text { Liljegren \& Osvalder (2004) } \\
\text { Ginsburg (2005) }\end{array}$ & Cook \& Woods (1996) \\
\hline The User & Kjeldskov \& Slov (2007) ${ }^{[17]}$ & $\begin{array}{l}\text { Xiao \& Seagull (2006) } \\
\text { Saleem }(2005)^{[28]}\end{array}$ \\
\hline The Work Context & Kushniruk et al. (2005) ${ }^{[15]}$ & $\begin{array}{l}\text { Patterson et al. (2002) } \\
\text { Nemeth et al. (2006) }\end{array}$ \\
\hline Theory & $\begin{array}{l}\text { Johnson et al. }(2005)^{[16]} \\
\text { Jaspers (2009) }\end{array}$ & $\begin{array}{l}\text { Effken (2006) } \\
\text { Guerlain (1999) }\end{array}$ \\
\hline Human Error & Koppel et al. (2005) ${ }^{[14]}$ & $\operatorname{Cook}(2006){ }^{[34]}$ \\
\hline
\end{tabular}

considered in each case, because of differences in their research goals. Usability researchers focus on physical, perceptual, and cognitive similarities and differences between users in order to design technology that supports all kinds of people. The user is an evaluator of the technology and may also be involved in the design process on a participatory basis. On the other hand, CSE researchers focus on the adaptive and resilient behaviors of users as they tailor their work practices and work environment (including technology) to achieve successful performance. Each design is considered a hypothesis about how technology shapes work behavior and it is the user who completes the design with their workarounds and tailor-made adaptations [43].

The Work Context. Usability and CSE researchers handle work context differently. In much usability evaluation, users are tested on specific tasks in controlled environments [44] where there is a strong focus on measurement. Contextual factors such as time and place, social norms or power structure are only considered later, in terms of how they affect the adoption and use of technology in the workplace. On the other hand, in CSE research there is less separation of work context, work itself and the technology designed to support work. CSE empirical work is usually conducted in the field where researchers explore the so-called 'wrapped package' of dynamic interactions among workers, tools, tasks and structures that make up the cognitive work context [45]. Consequently, CSE researchers may identify more subtle or tacit factors that should also be considered when evaluating designs, even though such factors may be less directly measurable.

Theory. The role of theory also differs across usability and CSE research. Usability and user testing are often embedded in the software engineering process. Designers may draw from various theories in cognitive science, cognitive psychology, and social psychology when developing and testing designs [46]. Similarly, evaluators may draw from theories of human perception and cognition when considering how a user might 
interact with a system. On the other hand, CSE involves the development of general theories about human interaction with systems, but particularly with technologyintensive and safety-critical systems. CSE research is informed by a broad range of theories such as systems theory, control theory, ecological psychology and cognitive sciences in the quest to gain insight into the interaction between people and technology. Model-development is a key theme.

Human error. Both the usability and CSE communities recognise that accidents conventionally attributed to 'human error' are more often the result of badly designed technology. However, there are differences in the way the two communities approach this problem. Usability researchers focus on how to design technology that reduces the likelihood of user mistakes, slips, and lapses. In contrast, CSE researchers see "error" as emerging from the same work processes that lead to success. People adapt their behavior to respond to workplace pressures towards efficiency or thoroughness [47]. Most of the time such adaptation is successful, but occasionally conditions are hostile and unintended consequences occur.

\section{Discussion and Conclusion}

The aim of this paper was to review research that demonstrates similarities and differences between CSE and usability-two research communities contributing to the design and evaluation of health information systems. Both communities share the goal of designing technologies that support human cognitive performance, and both encourage active user participation in the design and evaluation of health information systems. Although the review is limited in scope given our exclusion criteria, we hope it provides readers with a better understanding of the type of work carried out in both research communities and, more importantly, of some key distinctions between them.

The design and evaluation of human-centered health information systems is a challenging task. Reviews such as Greenhalgh et al.'s [2], Hoffman and Militello's survey of communities of practice investigating cognitive tasks [3], and the current paper can provide useful information to help healthcare researchers and practitioners make more informed decisions about which theoretical approaches and methods are most appropriate for their needs. Usability techniques can be very effective when practitioners need objective measurements about relatively fine points of interaction. However, researchers may wish to start with CSE methods when a broad perspective on work processes is needed, or if they want to check whether technology affects other work processes that are important for successful performance, but that are removed from the immediate point of interaction. All research communities have their unique strengths and limitations. It is up to the researcher to find the right combination that will help them answer their specific research question.

\section{Acknowledgments}

We gratefully acknowledge substantial input and assistance from members of the National Information and Communication Technology Australia (NICTA) Cognitive and Organisational Systems Engineering (COSE) project. NICTA is funded by the 
Australian Government as represented by the Department of Broadband, Communications and the Digital Economy and the Australian Research Council through the ICT Centre of Excellence program.

\section{References}

1. Wears, R., Berg, M.: Computer Technology and Clinical Work Still Waiting for Godot. Jama-Journal of the American Medical Association 293(10), 1261-1263 (2005)

2. Greenhalgh, T., et al.: Tensions and Paradoxes in Electronic Patient Record Research: A Systematic Literature Review Using the Meta-narrative Method. Milbank Quarterly 87(4), 729-788 (2009)

3. Hoffman, R.R., Militello, L.G.: Perspectives on cognitive task analysis. CRC Press, New York (2008)

4. ISO9241-11, Ergonomic requirements for office work with visual display terminals (VDTs) Part 11: Guidance on Usability (1998)

5. Dumas, J.: The great leap forward: The birth of the usability profession (1988-1993). Journal of Usability Studies 2, 54-60 (2007)

6. Nielsen, J.: Usability Engineering. Morgan Kaufmann, San Diego (1993)

7. Nielsen, J.: Heuristic Evaluation. In: Nielsen, J., Mack, R. (eds.) Usability Inspection Methods. John Wiley \& Sons, New York (1994)

8. Norman, D.A., Draper, S.: User-centred system design: New perspective on humancomputer interaction. Erlbaum Associates, Hillsdale (1986)

9. Polson, P.G., et al.: Cognitive walkthroughs - A method for theory-based evaluation of user interfaces. International Journal of Man-Machine Studies 36(5), 741-773 (1992)

10. Johnson, C.W.: Why did that happen? Exploring the proliferation of barely usable software in healthcare systems. Quality \& Safety in Health Care 15, I76-I81 (2006)

11. Allwood, C.M., Kalen, T.: User-Competence and other Usability Aspects when introducing a Patient Administrative System - A case-study. Interacting with Computers 5(2), 167-191 (1993)

12. Ash, J.S., Berg, M., Coiera, E.: Some unintended consequences of information technology in health care: The nature of patient care information system-related errors. Journal of the American Medical Informatics Association 11(2), 104-112 (2004)

13. Borycki, E., Kushniruk, A.: Identifying and preventing technology-induced error using simulations: Application of usability engineering techniques. Healthcare Quarterly 8, 99-105 (2005)

14. Koppel, R., et al.: Role of Computerized Physician Order Entry Systems in Facilitating Medication Errors. Jama-Journal of the American Medical Association 293(10), 11971203 (2005)

15. Kushniruk, A., et al.: Technology induced error and usability: The relationship between usability problems and prescription errors when using a handheld application. International Journal of Medical Informatics 74(7-8), 519-526 (2005)

16. Johnson, C.M., Johnson, T.R., Zhang, J.J.: A user-centered framework for redesigning health care interfaces. Journal of Biomedical Informatics 38(1), 75-87 (2005)

17. Kjeldskov, J., Skov, M.B.: Exploring context-awareness for ubiquitous computing in the healthcare domain. Personal and Ubiquitous Computing 11(7), 549-562 (2007)

18. Ginsburg, G.: Human factors engineering: A tool for medical device evaluation in hospital procurement decision-making. Journal of Biomedical Informatics 38(3), 213-219 (2005) 
19. Liljegren, E., Osvalder, A.L.: Cognitive engineering methods as usability evaluation tools for medical equipment. International Journal of Industrial Ergonomics 34(1), 49-62 (2004)

20. Jaspers, M.W.M.: A comparison of usability methods for testing interactive health technologies: Methodological aspects and empirical evidence. International Journal of Medical Informatics 78(5), 340-353 (2009)

21. Hollnagel, E., Woods, D.D.: Cognitive systems engineering - New wine in new bottles. International Journal of Man-Machine Studies 18(6), 583-600 (1983)

22. Rasmussen, J., Pejtersen, A., Goodstein, L.: Cognitive systems engineering. J. Wiley \& Sons, New York (1994)

23. Vicente, K.: Cognitive work analysis: Toward safe, productive, and healthy computerbased work. Lawrence Erlbaum Associates, Mahwah (1999)

24. Gaba, D.M., Deanda, A.: The Response of Anesthesia Trainees to Simulated Critical Incidents. Anesthesia and Analgesia 68(4), 444-451 (1989)

25. Institute of Medicine: Crossing the quality chasm: A new health system for the 21 st century. National Academy Press, Washington (2001)

26. Vicente, K.J.: Less is (sometimes) more in cognitive engineering: the role of automation technology in improving patient safety. Quality \& Safety in Health Care 12(4), 291-294 (2003)

27. Patterson, E.S., Cook, R.I., Render, M.L.: Improving patient safety by identifying side effects from introducing bar coding in medication administration. Journal of the American Medical Informatics Association 9(5), 540-553 (2002)

28. Saleem, J.J., et al.: Exploring barriers and facilitators to the use of computerized clinical reminders. Journal of the American Medical Informatics Association 12(4), 438-447 (2005)

29. Nemeth, C., et al.: Discovering Healthcare cognition: The use of cognitive artifacts to reveal cognitive work. Organization Studies 27(7), 1011-1035 (2006)

30. Xiao, Y., Seagull, F.J.: Emergent CSCW systems: The resolution and bandwidth of workplaces. International Journal of Medical Informatics (2006)

31. Guerlain, S., et al.: Interactive Critiquing as a Form of Decision Support: An Empirical Evaluation. Human Factors 41(1), 72-89 (1999)

32. Effken, J.A.: Improving clinical decision making through ecological interfaces. Ecological Psychology 18(4), 283-318 (2006)

33. Cook, R.I., Woods, D.D.: Implications of automation surprises in aviation for the future of total intravenous anesthesia (TIVA). Journal of Clinical Anesthesia 8, S29-S37 (1996)

34. Cook, R.: Being bumpable: Consequences of resource saturation and near-saturation for cognitive demands on ICU practitioners. In: Woods, D., Hollnagel, E. (eds.) Joint Cognitive Systems: Patterns in Cognitive Systems Engineering, pp. 23-35. Taylor \& Francis, London (2006)

35. Hollnagel, E., Woods, D., Leveson, N.: Resilience Engineering: Concepts and Precepts. Ashgate Publishing Company, Aldershot (2006)

36. Vicente, K.: Cognitive Engineering research at Riso from 1962-1979. In: Salas, E. (ed.) Advances in Human Performance and Cognitive Engineering Research, pp. 1-57. Elsevier, New York (2001)

37. Norman, D., Draper, S. (eds.): User Centered System Design - New Perspectives on Human-Computer Interaction. Lawrence Erlbaum Associates, London (1986)

38. Norman, D.A.: Cognitive engineering and education. In: Tum, D., Reif, F. (eds.) Problem Solving and Education: Issues in Teaching and Research, Erlbaum Associates, Hillsdale (1980)

39. Norman, D.: The Psychology of Everyday Things. Basic Books, New York (1988) 
40. Gray, W.D.: Cognitive architectures: Choreographing the dance of mental operations with the task environment. Human Factors 50(3), 497-505 (2008)

41. Benyon, D., Turner, P., Turner, S.: Designing interactive systems: People, activities, contexts, and technologies. Person Education Limited, Harlow (2005)

42. Sarter, N., Woods, D., Billings, C.: Automation Surprise. In: Salvendy, G. (ed.) Handbook of Human Factors and Ergonomics. Wiley \& Sons, New York (1997)

43. Woods, D.D.: Designs are hypotheses about how artifacts shape cognition and collaboration. Ergonomics 41, 168-173 (1998)

44. Dumas, J., Redish, J.: A practical guide to usability testing. Intellect Books, Exeter (1999)

45. Hoffman, R., Militello, L.: Perspectives on Cognitive Task Analysis: Historical Origins and Modern Communities of Practice. Psychology Press, New York (2008)

46. Carroll, J.: HCI models, theories, and frameworks: toward a multydisciplinary science. Morgan Kaufmann, Amsterdam (2003)

47. Hollnagel, E.: ETTO principle: Efficiency-thoroughness tradeoff - Why things that go right sometimes go wrong. Ashgate Publishing Ltd., Farnham (2009) 